ther by the month or anus, appear to con-! what similar case (which may be sketched sist of dark-coloured blood, poured out by hereafter), I boldly attempted the poor the vessels of the villous cont of the sto-' man's relief, under feelings of considerable mach and intestines whilst in a state of 'responsibility, and before a crowd of specexcitement, with an admixture of vitiated tators. I washed the mass of bowels with bile, gastric and intestinal secretions.

Dublin, No..2, Blessington St., Feb. 10, 1832. some warm potats water (the bits of boiled roots 1 knew were not hurtful). I applied general pressure, hoping to force up some of the contents; but this was soon given up. Then relaxing the abdomen by position, I began, by minute gradations, to

\section{SUDDEN EXPULSION OF THE BOWELS PER ANUM.}

\section{To the Editor of THE LanceT.}

Sin,-The following case appears to me deserving of publicity, and should you be of the same opinion, you will have the goodness to give it a place in your admirable Journal. I am, Sir, yours,

T. Dickson, Surg., R.N.

Cookstown, Co. Tyrone, Ireland, Feb. 1, 1832.

Nov. 12, 1331, about ten P.II., I was summoned in the utmost haste, and with most earnest supplications, by a young man, to see his father Putrick Gilman, aged about 55, of fair complexion, stout frume, and good constitution, a mason by trade, who had, to all appearance, met with his death. I found him lying in the kitchen of a poor cottage in the suburbs, almost insensible, retaining some power of speech, but that incoherent. It appeared that he had drunk a great quantity of beer, being fair day; that late in the day he had been attacked by diarrhœa and tenesmus, and that in one of his attempts to relieve nature, he had fallen backwards from an eminence, on to the pavement. Immediately he exclaimed " he was gone," becoming suddenly sick and faint. A blanket had been thrown over him, and one or two respectable practitioners, who bad examined his situation, were standing aloof, appearing disposed to leave him to his fate, which disposition I could not disapprove of, when, on raising the blanket, the whole of the small intestines, of extent sufficient to fill a peck measure, appeared outside the anus, fouled with muil, gravel, and chaff. The ileum was pushed back over the sacrum in four or five large curves, inflated, stiff, cold, and brown; the mesentery stretched six inches beyond the anus; the jejunum towards the perinæum, in several smaller folds, of a light carnation, softer and warmer. The pulse was indistinct, and the whole surface cadaverous, both in as pect and to the feel.

Encouraged by the recollection of a somereturn that part of the jejurium which had protruded last; but after getting up a few inches, they were suddenly expelled by spasms, accompanied by heavy groans. "I then got him held up on his knees and breast, and renewing my efforts, gradatim, I found it, after some time, going up, and finally, in about ten minutes (from raising him on his knees), the whole was replaced. About twenty minutes had been already expended in attempts in the horizontal position. I thought 1 felt it return through an irregular opening in the fore part of the rectum next the bladder, but, it may be supposed, 1 did not minutely examine it. $\boldsymbol{A}$ firm compress and $\mathrm{T}$ bandage were then applied, the patient carried to his own house, put to bed under a vigilant watch, and two grains of opium administered.

Next mornin found lim rational; pulse full and strung, not very frequent; some liquid had escaped from the anus; the compress and bandage giving great annoyance, were removed for the day, and had no occasion to be reapplied. He had frequent colic pains, repeated vomiting, great thirst, and universal prostration. He was immediately bled to ijlbs.; had fomentations applied to the abdomen, and strict antipblogistic regimen enjoined; nothing allowed but barley water with a little nitre and cream of tartar in it. Next day (14th) felt better, but the vomiting continued; bowels constipated. A drachm of sulphate of magnesia, dis. solved in cold water, was given every third hour; warm fomentations occasionally.

15. Vomiting ceased; colic pains abated, and bowels opened; no tension of the abdomen; pulse nearly natural. He was restricted to farinaceous slops for 10 days longer, and gradually recovered, taking nothing excepting a little rhubarb occasionally, and one bottle of infusion of gentian. In a month he was at work, as well as ever, and so contivues.

P.S. He laboured under scrotal hernia of the right side; it was never returned, ap. peared entirely omental, gave him no trouble, and was evidentiy unconnected with the accident.

Cookstown Dispensary. 